RASĀYAN J. Chem.

Vol. 14 | No. 2 |855-860| April - June | 2021

ISSN: 0974-1496 | e-ISSN: 0976-0083 | CODEN: RJCABP

http://www.rasayanjournal.com

http://www.rasayanjournal.co.in

\title{
SELECTIVE SYNTHESIS OF BENZALDEHYDES BY PERMANGANATE OXIDATION OF BENZYL ALCOHOLS IN NON-POLAR SOLVENTS UNDER PHASE TRANSFER CATALYSIS
}

\author{
P. Bashpa ${ }^{1}$, C. Kavitha ${ }^{2}$ and K. Bijudas ${ }^{1, \bowtie}$ \\ ${ }^{1}$ Department of Chemistry, N. S. S. College, Manjeri, Malappuram-676122, (Kerala), India \\ ${ }^{2}$ Department of Chemistry, Fathima Arts \& Science College, Moothedam, Nilambur, \\ Malappuram-679331, (Kerala), India \\ ${ }^{\square}$ Corresponding Author: bijudask@gmail.com
}

\begin{abstract}
The reported study deals with the selective synthesis of benzaldehyde and substituted benzaldehydes by the permanganate oxidation of benzyl alcohols under phase transfer catalysis in non-polar solvents. This selective oxidation of benzyl alcohols with phase transferred permanganate was studied in a two-phase system at $30^{\circ} \mathrm{C}$. The phase transfer catalysts used were quaternary ammonium and phosphonium salts with ethyl acetate and toluene as the solvents. Permanganate ion was transferred into the organic phase with the help of phase transfer catalysts and able to oxidize benzyl alcohols to corresponding benzaldehydes even in the absence of mineral acids. The reaction was found to be highly selective since no formation of benzoic acid or substituted benzoic acids were detected and the yield of corresponding benzaldehydes was found to be more than $90 \%$. The products obtained were precipitated as 2,4-dinitrophenylhydrazone and the recrystallized products were analyzed by melting point and by spectroscopic techniques like infra-red and UV-visible analyses. The stoichiometry of the oxidation was established as two moles of permanganate were found to be equivalent to three moles of benzyl alcohols. A suitable reaction path according to the observations is proposed with Stark's phase transfer catalytic cycle.
\end{abstract}

Keywords: Benzyl Alcohols, Benzaldehydes, Phase Transfer Catalysis, Phase Transfer Catalyst, Permanganate Ion, Selective Synthesis, Non-polar Solvents

RASĀYAN J. Chem., Vol. 14, No.2, 2021

\section{INTRODUCTION}

Different oxidizing agents were employed in various oxidation reactions and have great significance in the synthesis of organic compounds of industrial importance. ${ }^{1,2}$ Various oxidants like permanganate, chromate, hypochlorite, hydrogen peroxide etc. were used commonly in many oxidation transformations. Permanganate is a strong, inexpensive oxidant that is widely used in organic synthesis. ${ }^{3-6}$ Since most of the organic compounds are soluble only in non-polar solvents, the application of permanganate ion as an oxidant is limited in organic synthesis. This is the case in most of the common oxidants used nowadays. The introduction of phase transfer catalysis (PTC) has brought dramatic changes in organic synthesis by enhancing the solubility of anions in non-polar solvents with their ability to catalyze the reaction. PTC is one of the most efficient methods that can be used to carry out various chemical reactions under mild conditions with improved results. Application of PTC in organic synthesis provides cost reduction and pollution prevention which is highly significant in the era of green chemistry.$^{7-9}$ Phase transfer catalysts (PT catalysts) transfer the reactive permanganate ions from the aqueous phase to the organic phase and effecting the reaction in the organic medium with ease. PTC method involves the transfer of inorganic anions from the aqueous medium into the non-polar organic medium in the form of an ion-pair with the cationic moiety of PT catalyst having appropriate size and lipophilicity. The anions thus transferred and poorly solvated in the organic medium is found to exhibit greater reactivity. This enables the substrate to react faster in the non-polar medium in the presence of a PT catalyst. The reaction can be carried out in a biphasic system involving an inexpensive non-polar aprotic solvent and water without using highly polar solvents. The commonly used PT catalysts used are crown ethers and quaternary onium salts in which the 
RASĀYAN J. Chem.

Vol. 14 | No. 2 |855-860| April - June | 2021

quaternary onium salts are more suitable in selectivity, ease of use and low cost. ${ }^{10,11}$ Even a small quantity of PT catalyst can transfer inorganic oxidant from the aqueous phase to the organic phase in the form of an ion pair. The active oxidant as ion pair in the organic phase interacts with the substrate forming the product with the enhanced rate at optimum conditions. There are reports on using permanganate, chromate, hypochlorite etc. as an oxidizing agent in organic synthesis under PTC for the oxidation of various organic compounds. ${ }^{12-19}$ But reports on the selective oxidation of benzyl alcohols to corresponding benzaldehydes using permanganate under PTC is scanty and hence the present work is reported. Even though there are reports on similar work, the PT catalysts used were crown ethers which are inferior to quaternary onium salts in terms of selectivity, availability, purity, cost and reactivity. ${ }^{20,21}$ Permanganate ion is considered a strong oxidizing agent and it is very difficult to give selectivity in product formation since further oxidation cannot be prevented in normal conditions. The uniqueness of the reported work is that benzyl alcohols were selectively oxidized to corresponding benzaldehydes and no traces of benzoic acid were detected at the reported experimental conditions. This shows the selectiveness and mildness of permanganate ion under PTC which can be considered as a unique character of permanganate in oxidation reactions.

This paper reports the selective oxidation of benzyl alcohol and its para-substituted derivatives using various quaternary phosphonium and ammonium salts as PT catalysts in various organic solvents namely ethyl acetate and toluene by permanganate. These reactions were carried out at $30^{\circ} \mathrm{C}$ by simple stirring on a magnetic stirrer for half an hour carried in greener solvents like ethyl acetate and toluene. The substituted benzyl alcohols used were 4-hydroxy benzyl alcohol, 3-hydroxy benzyl alcohol, 4chlorobenzyl alcohol, 3-chlorobenzyl alcohol, 4-methoxybenzyl alcohol, 3-methoxybenzyl alcohol, 4nitrobenzyl alcohol, 3-nitrobenzyl alcohol and 4-methylbenzyl alcohol. The PT catalysts used were tetrabutylammonium bromide (TBAB), tetrabutylphosphonium bromide (TBPB), tetrabutylammonium hydrogen sulphate (TBAHS) and cetyltrimethylammonium bromide (CTMAB).

\section{Material and Methods}

\section{EXPERIMENTAL}

Analar grade potassium permanganate (Merck, India) was used and its solution was prepared in doubly distilled water. Benzyl alcohol (Merck, India, AR) was further purified by distillation under reduced pressure. The substituted benzyl alcohols, 4-hydroxybenzyl alcohol, 3-hydroxybenzyl alcohol, 4-chlorobenzyl alcohol, 3-chlorobenzyl alcohol, 4-methoxybenzyl alcohol, 3-methoxybenzyl alcohol, 4nitrobenzyl alcohol, 3-nitrobenzyl alcohol and 4-methylbenzyl alcohol (Spectrochem, India Ltd. Mumbai and Merck KGaA, Germany) were used as such. Tetrabutylammonium bromide (TBAB), tetrabutylammonium hydrogen sulphate (TBAHS) and cetyltrimethylammonium bromide (CTMAB) (Spectrochem India Ltd. Mumbai) and tetrbutylphosphonium bromide (Merck KGaA, Germany) were used as PT catalyst. The organic solvents toluene and ethyl acetate were purified according to the standard procedure. ${ }^{22,23}$ The purified solvents were refluxed for 1-2 hours with a mixture of PT catalyst and potassium permanganate and then distilled.

\section{General Procedure}

Synthetic analysis was carried out in a heterogeneous fashion under room temperature of $30^{\circ} \mathrm{C}$. Benzyl alcohol and substituted benzyl alcohols $(0.1 \mathrm{~mol})$ were dissolved in $50 \mathrm{~mL}$ toluene or ethyl acetate which contains $0.01 \mathrm{~mol}$ PT catalyst was mixed with $50 \mathrm{~mL}(0.5 \mathrm{~mol})$ potassium permanganate. The mixture was stirred vigorously using a magnetic stirrer for about thirty minutes at $30^{\circ} \mathrm{C}$. The organic layer was extracted with ether three times. This organic layer was again extracted with $10 \%$ sodium bicarbonate and both organic and aqueous layers were separated. A saturated solution of 2,4-dinitrophenylhydrazine in $\mathrm{HCl}$ was added to the organic layer and kept overnight in a refrigerator. The precipitated 2,4-dinitrophenylhydrazone (DNP) was filtered off, recrystallized from ethanol, dried and weighed. The same procedure was repeated for all the substrates in both solvents. The products were analyzed with their melting point and other qualitative spectral techniques like UV-visible spectroscopy and infra-red spectroscopy. The aqueous layer after extraction with sodium bicarbonate was acidified with concentrated $\mathrm{HCl}$ to detect the presence of acid. The stoichiometry of the reaction was determined by mixing a known 
RASĀYAN J. Chem.

Vol. 14 | No. 2 |855-860| April - June | 2021

quantity of benzyl alcohol with a known excess quantity of phase transferred permanganate and by estimating the [permanganate] remaining after completion of the reaction.

\section{RESULTS AND DISCUSSION}

The stoichiometry of the oxidation was established by the equilibrating known excess concentration of the phase transferred permanganate with a known amount of the corresponding alcohol. Two moles of permanganate were found to be equivalent to three moles of benzyl alcohols.

$$
3 \mathrm{PhCH}_{2} \mathrm{OH}+2 \mathrm{Q}^{+} \mathrm{MnO}_{4}^{-} \rightarrow 3 \mathrm{PhCHO}+2 \mathrm{Q}^{+}(\mathrm{OH})^{-}+2 \mathrm{H}_{2} \mathrm{O}
$$

All the substrates (benzyl alcohol and substituted benzyl alcohols) on oxidation with permanganate under PTC in non-polar solvents at room temperature gave corresponding aldehydes as the product. The yield was found to be very high (above 90\%) and under the experimental conditions and there is no further oxidation of the aldehyde to acid even after stirring for three hours. This is evident from the absence of benzoic acid or substituted benzoic acids in the reaction mixture. The recrystallized sample of products in the form of 2,4-dinitrophenylhydrazone was characterized by its melting point and is given in Table-1.

Table-1: Melting Point of Products in the form of 2,4-DNP on oxidation of Benzyl Alcohol and substituted Benzyl Alcohols

\begin{tabular}{c|l|c}
\hline S. No. & \multicolumn{1}{|c|}{ Substrate } & $\begin{array}{c}\text { Melting point of DNP } \\
\text { of product }\left({ }^{0} \mathrm{C}\right)\end{array}$ \\
\hline 1 & Benzyl alcohol & $239 \pm 2$ \\
\hline 2 & 4-chlorobenzyl alcohol & $255 \pm 1$ \\
\hline 3 & 3-chlorobenzyl alcohol & $255 \pm 1$ \\
\hline 4 & 4-nitrobenzyl alcohol & $320 \pm 2$ \\
\hline 5 & 3-nitrobenzyl alcohol & $290 \pm 1$ \\
\hline 6 & 4-hydroxybenzyl alcohol & $272 \pm 2$ \\
\hline 7 & 3-hydroxybenzyl alcohol & $255 \pm 2$ \\
\hline 8 & 4-methylbenzyl alcohol & $233 \pm 2$ \\
\hline 9 & 4-methoxybenzyl alcohol & $252 \pm 1$ \\
\hline 10 & 3-methoxybenzyl alcohol & $253 \pm 1$ \\
\hline
\end{tabular}

The above results were compared with the reported values of authentic samples and found to show excellent similarity proving the formation of corresponding aldehydes. This was further confirmed by infra-red and UV- visible spectral techniques by taking benzyl alcohol as the typical substrate. The infrared absorption spectrum of the 2,4-DNP of the obtained product was recorded from $\mathrm{KBr}$ pellets using Jasco FT-IR 4100 spectrophotometer (Japan) and is shown in Fig.-1. The infra-red spectrum showed peaks at $3065 \mathrm{~cm}^{-1}$ (aromatic C-H stretching), $2820 \mathrm{~cm}^{-1}$ (aldehydic C-H stretching, asymmetric), $2740 \mathrm{~cm}^{-1}$ (aldehydic C-H stretching, symmetric), $1700 \mathrm{~cm}^{-1}$ (C=O stretching), $3065 \mathrm{~cm}^{-1}$ (aromatic $\mathrm{C}-\mathrm{H}$ stretching), $1490 \mathrm{~cm}^{-1}$ and $1600 \mathrm{~cm}^{-1}(\mathrm{C}=\mathrm{C}$ stretching in aromatic ring). The presence of these specified peaks leads to the conclusion that the product formed may be benzaldehyde. Furthermore, this spectrum was compared with that of pure benzaldehyde and found to have excellent similarities.

UV-Visible absorption of the product in the form of 2,4-DNP on the oxidation of benzyl alcohol was recorded by Hitachi U-3000 UV-Visible spectrophotometer using $1 \mathrm{~cm}$ quartz cell and Spectro grade ethanol (Merck, India) as a solvent and is given in Fig.-2. The UV-Visible spectrum of 2,4-DNP of the product showed intense peaks at $235 \mathrm{~nm}$ and $353 \mathrm{~nm}$ which were assigned to $\pi-\pi^{*}$ and $\mathrm{n}-\pi^{*}$ transition of the aromatic compound. The UV-Visible spectrum of the obtained product was compared with that of 2,4-DNP of pure benzaldehyde and gave excellent similarities.

The selective oxidation process of benzyl alcohols by phase transferred permanganate was followed by spectrophotometrically to find out the reaction pathway. The presence of isosbestic points at $498 \mathrm{~nm}$ and $582 \mathrm{~nm}$ in the sequential scan of the absorption spectrum of the reaction mixture proved the formation of 
RASĀYAN J. Chem.

Vol. 14 | No. 2 |855-860| April - June | 2021

a single product with a similar reaction path and rules out a complicated sequence of events during the reaction. ${ }^{24,25}$

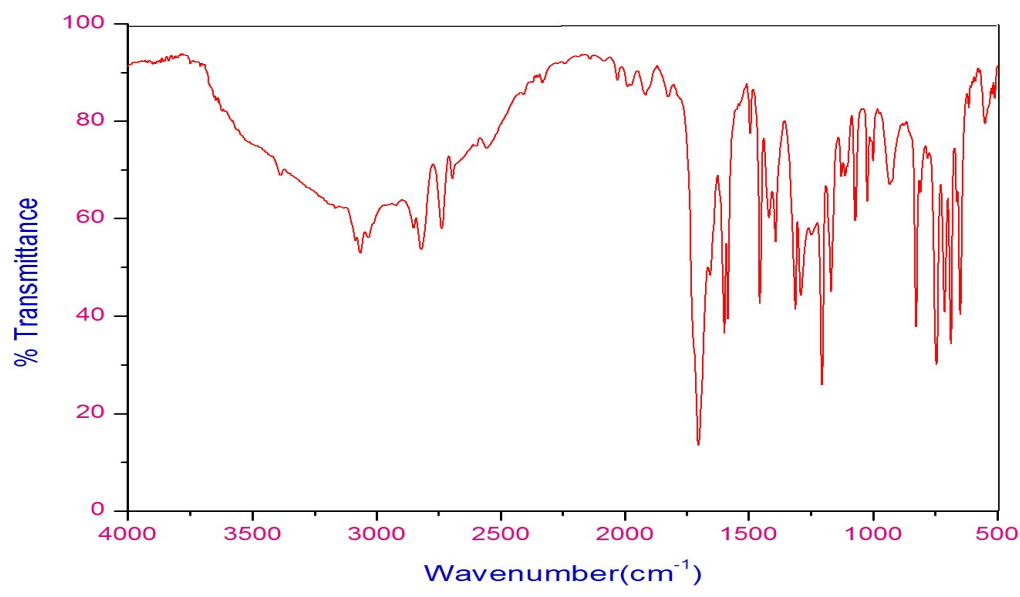

Fig.-1: Infra-red Spectrum of 2,4-dinitrophenylhydrazone of Product

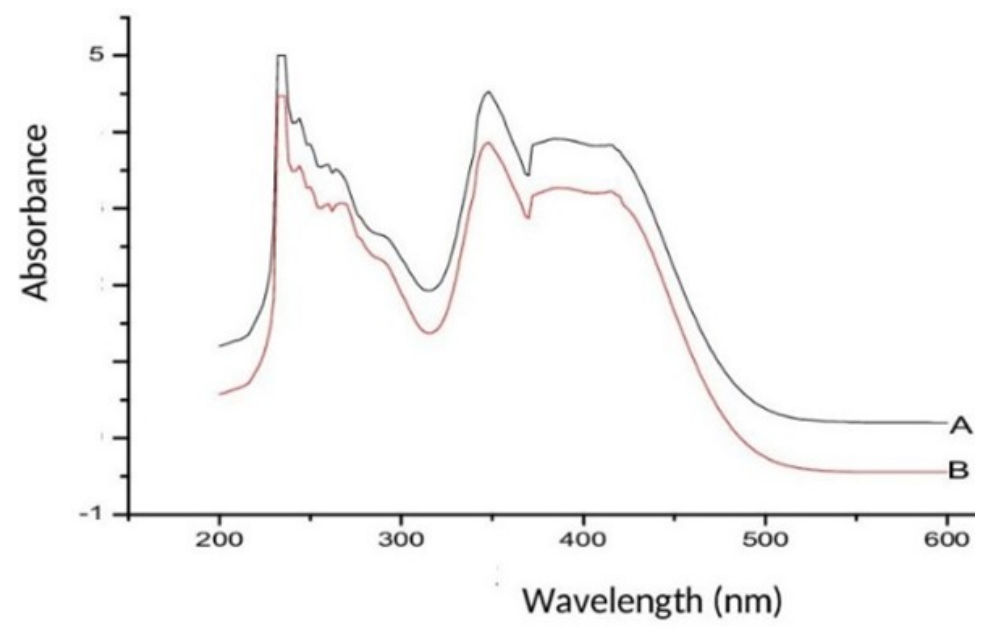

Fig.-2: UV-Visible Spectrum of 2,4-dinitrophenylhydrazone of pure benzaldehyde(A) and 2,4-dinitrophenylhydrazone of product on the oxidation of benzyl alcohol (B)

Selective oxidation of benzyl alcohol and its para-substituted derivatives by permanganate ions in nonpolar solvents under phase transfer catalysis gave corresponding benzaldehydes. This simple process is found to be highly selective since there are no traces of acids and benzaldehyde and substituted benzaldehydes were formed with high yield and purity. All the PT catalysts used in the oxidation functioned well in their catalytic activity, but the yield and ease of reaction are slightly different as in the order TCMAC $>$ TBPB $>$ TBAB $>$ TBAHS $>$ CTMAB. This is on account of changes in the combination of alkyl or aryl groups and also may be due to the differences in the activity of anions for phase transfer. The cationic part of PT catalyst requires that they collectively should have sufficient organic structure to transfer the desired anion into the organic phase. Thus, PT catalysts having larger alkyl or aryl groups with an almost symmetric structure are most effective both in anion transfer and in reactivity. Therefore, TCMAC with more bulky and symmetrical alkyl groups has higher reactivity in comparison with other PT catalysts used. Even though both TBPB and TBAB have the same alkyl part, phosphonium ion is always a better central onium atom with more reactivity than ammonium and hence TBPB gives a higher 
RASĀYAN J. Chem.

Vol. 14 | No. 2 |855-860| April - June | 2021

yield than TBAB. Both TBAHS and CTMAB also function as good PT catalysts but there is a tendency to form an emulsion in most of the reactions involving these catalysts. The reaction is very simple and fast in both ethyl acetate and toluene, but the yield of the product is found to be slightly more in ethyl acetate than that in toluene. The solubility and partitioning behaviour of the quaternary salts are markedly affected by slight changes like the organic phase. Solubility and partitioning of quaternary onium salts are increased by increasing the polarity of the aprotic organic phase. Ethyl acetate is more polar than toluene and hence ethyl acetate is a better solvent than toluene in terms of ease of reaction, better yield and consideration as a green solvent. Based on the experimental results obtained, a possible Stark's phase transfer catalytic cycle for the oxidation of benzyl alcohols by permanganate ions is given in Scheme-1 and a possible reaction path is given in Scheme-2.

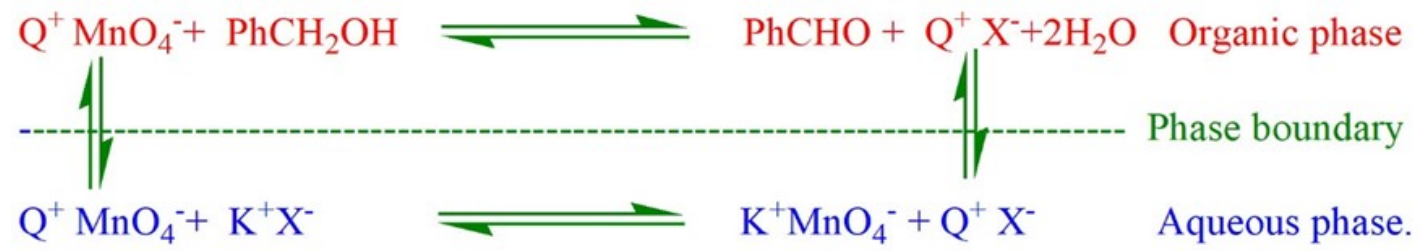

Scheme-1: Stark's Catalytic Cycle for the Oxidation of Benzyl Alcohol by Permanganate

$$
\begin{aligned}
& \mathrm{KMnO}_{4} \rightleftharpoons \mathrm{K}^{+}+\mathrm{MnO}_{4}^{-} \\
& \left(\mathrm{Q}^{+} \mathrm{X}^{-}\right)_{\mathrm{aq}}+\mathrm{MnO}_{4}^{-} \rightleftharpoons\left(\mathrm{Q}^{+} \mathrm{MnO}_{4}^{-}\right) \mathrm{aq}^{+} \mathrm{X}^{-} \mathrm{aq} \\
& \left(\mathrm{Q}^{+} \mathrm{MnO}_{4}^{-}\right)_{\mathrm{aq}} \rightleftharpoons\left(\mathrm{Q}^{+} \mathrm{MnO}_{4}^{-}\right)_{\mathrm{org}} \\
& \mathrm{PhCH}_{2} \mathrm{OH}+\left(\mathrm{Q}^{+} \mathrm{MnO}_{4}^{-}\right)_{\text {org }} \rightleftharpoons \mathrm{PhCHO}+\left(\mathrm{Q}^{+} \mathrm{X}^{-}\right) \text {org } \\
& \left(\mathrm{Q}^{+} \mathrm{X}^{-}\right)_{\text {org }} \rightleftharpoons\left(\mathrm{Q}^{+} \mathrm{X}^{-}\right) \mathrm{aq}
\end{aligned}
$$

Scheme-2: Reaction Path for the Oxidation of Benzyl Alcohols by Permanganate

\section{CONCLUSION}

In summary, a simple, efficient, selective green method for the conversion of benzyl alcohols to corresponding benzaldehydes under PTC was reported. Permanganate ions were used as the oxidant and the reaction was found to be highly selective and smooth with excellent yield. Greener solvents were used for this conversion and there was no formation of by-products. The method reported hereunder PTC is found to be very unique in selectivity, mildness, yield and ease of reaction especially permanganate ion is used as oxidant. This method can also be adapted to other reaction systems by which all the above mentioned qualities can be achieved and can be incorporated in chemical industries with the motto of cost reduction and pollution prevention.

\section{REFERENCES}

1. D.G. Lee, Oxidation of Organic Compounds by Permanganate Ion and Hexavalent Chromium, Open Court: La Salle 1980.

2. K.B. Wiberg, Oxidation in Organic Chemistry, Academic Press, London and New York 1965.

3. E.V. Dehmlow and S.S. Dehmlow, Phase transfer catalysis, VCH: Weinheim, Germany 1993.

4. D. Pletcher and S.J.D Tait, Tetrahedron Letters, 19, 18 (1978), DOI:10.1016/S0040-4039(01)94615-5

5. Y. Sasson and R. Neumann, Handbook of Phase Transfer Catalysis, Kluwer Academic Publishers, 
RASĀYAN J. Chem.

Vol. 14 | No. 2 |855-860| April - June | 2021

Dordrecht 1993.

6. C.M. Starks and C.L. Liotta, Phase transfer catalysis, Principles and Techniques, Academic Press, New York 1978.

7. K. Bijudas and T.D. Radhakrishnan Nair, Indian Journal of Chemistry, 43A, 6 (2004).

8. M. Mishra and B. Torok, Green Chemistry. An Inclusive Approach, Elsevier, p. 449-470, 2018.

9. G.D. Yadav and C.K. Mistry, Journal of Molecular Catalysis A., 172, 1(2001), DOI:10.1016/S1381$1169(01) 00132-7$

10. R.A. Jones, Quaternary Ammonium Salts, Their Use in Phase-Transfer Catalyzed Reactions, Academic Press, New York 2001.

11. F. Nathalia, Carvalho. and J.R. Pliego Jr., Journal of Organic Chemistry, 81, 18(2016), DOI: $10.1021 /$ acs.joc.6b01624

12. S. Abramovici, R. Neumann and Y. Sasson, Journal Molecular Catalysis 29, 3(1985), DOI: $10.1016 / 0304-5102(85) 80037-7$

13. K. Bijudas, P. Bashpa, V.P. Bipin L. Nair A.P. Priya, M. Aswathy, C. Krishnendu and P. Lisha, Bulletin of Chemical Reaction Engineering and Catalysis, 10, 1(2015), DOI: $10.9767 /$ bcrec.10.1.7189.38-42

14. G.A. Lee and H.H. Freedman, Israel Journal of Chemistry, 26, 3(1985), DOI: $10.1002 /$ ijch. 198500100

15. G.A. Mirafzal and A.M. Lozeva, Tetrahedron Letters, 39, 40(1998), DOI:10.1016/S00404039(98)01584-6

16. P. Rajendran, T. Divya, P. Bashpa and K. Bijudas, Journal of Chemical and Pharmaceutical Sciences, 1 (2016).

17. M.L Wang. and T.H Huang, Chemical Engineering Communications, 194, 5(2007), DOI: $10.1080 / 00986440600992685$

18. K. Bijudas, P. Bashpa and T.D. Radhakrishnan Nair, Bulletin of Chemical Reaction Engineering \& Catalysis, 9, 2(2014) DOI:10.9767/bcrec.9.2.6476.142-147

19. D.S. Rathore and C.P. Singh Chandel, Rasayan Journal of Chemistry, 13, 1(2020), DOI: $10.31788 /$ RJC.2020.1315612

20. N. Jose, S. Sengupta and J.K. Basu, Journal of Molecular Catalysis A, 309, 1(2009), DOI: 10.1016/j.molcata.2009.05.009

21. K.N. Rankin, Q. Liu, J. Hendry, H. Yee, N.A. Noureldin and D.G. Lee, Tetrahedron Letters, 39, 10(1998), DOI:10.1016/S0040-4039(97)10788-2

22. D.D. Perrin, W.L. Armarego and D. R. Perrin, Purification of Organic Compounds, Pergamon Press, Oxford 1966.

23. A.I. Vogel, Text Book of Practical Organic Chemistry, Longman, London 1967.

24. S. Dash and B.K. Mishra, Indian Journal of Chemistry, 36A, 8 (1997).

25. A. Fawzy, I.A. Zaafarany, I. Althagafi, J. Alfahemi and M. Morad, Austin Chemical Engineering, 3, 1(2016).

[RJC-6188/2020] 